\title{
Visibilidade dos pesquisadores no tema Estudos Métricos: análise de citação e co-citação nos periódicos do SciELO
}

\author{
Visibility of researchers in metric studies: a citation and co-citation analysis in SciELO
}

\author{
Maria Cláudia Cabrini Gracio (1), Ely Francina Tannuri OliveIRA (2), Gislaine I. de MAtos (3) \\ Faculdade de Filosofia e Ciências - Unesp, - Av. Hygino Muzzi Filho, 737 Marília - São Paulo - Brasil - \\ CEP: 17525-900. (1) cabrini@marilia.unesp.br (2) etannuri@flash.tv.br (3) gislaineisa@gmail.com
}

\begin{abstract}
Resumen
La propuesta de esta investigación es analizar y dar visibilidad al frente de investigación del tema Estudios Métricos, en el área de Ciencia de la Información, desde las citaciones y cocitaciones en los artículos de los periódicos publicados online por el SciELO, a saber: Ciencia de la Información y Perspectiva en Ciencia de la Información. Se partió de la consideración de que la frecuencia de las citaciones y cocitaciones retratan las relaciones establecidas entre los investigadores y el frente de investigación en el tema, considerando que las citaciones y cocitaciones presuponen investigaciones con similitudes de asunto y asociación o coocurrencia de ideas. Se trabajó, al principio, con el universo de 388 artículos, siendo 53 (13,7\%, entre los dos periódicos) los artículos que tratan del tema Estudios Métricos. Fueron examinados los autores más citados; en un total de 1514 citaciones, 35 autores fueron citados por lo menos 6 veces de un total de 318 citaciones. La matriz de cocitaciones se constituyó por una matriz simétrica, $18 \times 18$, con las cocitaciones planteando un primer frente de investigación. Los autores pioneros en estudios métricos componen el núcleo de este frente.
\end{abstract}

Palabras clave: Visibilidad de investigadores. Análisis de citación. Análisis de cocitación. Red social de cocitación.

\section{Introdução}

O desenvolvimento da ciência como sistema é governado pela produção, fluxo e disseminação do conhecimento. Esta última se faz através das publicações. Assim, o conhecimento científico fica disponibilizado para a comunidade e pode ser visualizado tanto no que se refere aos produtores como ao próprio produto da ciência produzida para retroalimentar o processo de comunicação científica.

Toda produção na ciência, qualquer que seja o campo, tem como premissa o fato de que o

\begin{abstract}
The proposal of this research is to analyze and attract public attention to the research front of the subject Metric Studies, in the Information Science area. Citations and co-citations were analyzed in articles of the periodicals published online by SciELO, namely: Ciência da Informação and Perspectiva em Ciência da Informação. It was estimated that the frequency of citations and co-citations shows the established relations between the researchers and the front of research in the subject, considering that the citations and cocitations show researches with subject similarities and ideas association or co-occurrence. It was used, initially, the universe of 388 articles; 53 (13.7\%, including the two periodicals mentioned) of them are about the subject Metric Studies. The cited authors were examined; in a total of 1514 citations, 35 authors had been cited at least 6 times in a total of 318 citations. The cocitation matrix consisted of a symmetrical matrix, $18 \times 18$, with co-citations delineating a first research front. The pioneering authors in metric studies compose the core of this front.
\end{abstract}

Keywords: Visibility of researchers. Citation analysis. Co-citation analysis. Co-citation social net.

cientista constrói seu trabalho a partir de trabaIhos anteriores, e mostra isso mencionando-os em uma lista de referências. Assim, no ato de citar, o pesquisador identifica os pesquisadores anteriores, cujos conceitos, métodos ou teorias serviram de paradigma e referência para seu trabalho atual, gerando uma listagem de citações.

Partiu-se da premissa de que a freqüência das citações e cocitações retrata as relações estabelecidas entre os pesquisadores e a frente de pesquisa no tema, considerando que as mesmas pressupõem pesquisas com similaridades 
de assunto e associação ou co-ocorrência de idéias.

Nesta pesquisa, focou-se o tema "Estudos métricos" na área de Ciência da Informação e tomou-se como fonte de referências as publicações dos periódicos do SciELO, biblioteca eletrônica considerada relevante pelas publicações de periódicos nucleares e consolidados nas diferentes áreas do conhecimento.

Nas últimas décadas, tanto no âmbito internacional como de Iberoamérica, os estudos sobre métricas na informação, especialmente Bibliometria e Cienciometria, vêm ganhando consistência. No Brasil, estudos sistemáticos sobre a evolução e tendência das ciências iniciaram-se nos anos 70 e vêm sendo consolidados por pesquisadores de diferentes áreas, especialmente da Ciência da Informação.

A proposta é utilizar recursos métricos, especialmente análise de citação e cocitação, na avaliação da produção científica no tema "Estudos métricos", tomando como fonte de referência as publicações dos periódicos do SciELO na área de Ciência da Informação, com a finalidade de visualizar a frente de pesquisa no tema em questão.

\section{Objetivos}

O objetivo é analisar e dar visibilidade à frente de pesquisa no tema Estudos Métricos, na área de Ciência da Informação, a partir das citações e cocitações dos artigos dos periódicos publicados on-line pelo SciELO, a saber: Ciência da Informação e Perspectiva em Ciência da Informação.

De modo mais específico, pretende-se, através da análise das referências dos autores em suas produções, destacar quais são os mais citados pelas revistas arroladas, bem como aqueles que foram cocitados de modo a destacar a frente de pesquisa no tema "Estudos Métricos".

\section{Referencial Teórico}

A Scientific Electronic Library Online - SciELO é uma biblioteca eletrônica que abrange uma coleção selecionada de periódicos científicos brasileiros e resultou de um projeto de pesquisa da Fundação de Amparo à Pesquisa do Estado de São Paulo (FAPESP), em parceria com o Centro Latino-Americano e do Caribe de Informação em Ciências da Saúde (BIREME). A partir de 2002, passou a contar com o apoio do Conselho Nacional de Desenvolvimento Científico e Tecnológico (CNPq).
Seu objetivo é o desenvolvimento de uma metodologia comum para a preparação, armazenamento, disseminação e avaliação da produção científica em formato eletrônico. Atualmente, através deste projeto, são publicados 204 periódicos, envolvendo oito campos de conhecimentos: Ciências Agrárias, Ciências Biológicas, Ciências da Saúde, Ciências Exatas e da Terra, Ciências Sociais e Aplicadas, Engenharias, Linguística, Letras e Artes. No campo Ciências Sociais e Aplicadas encontra-se a área Ciência da Informação, com publicações de dois periódicos: Perspectivas em Ciência da Informação e Ciência da Informação.

O primeiro, Perspectiva em Ciência da Informação, é uma publicação quadrimestral da Escola de Ciência da Informação da UFMG (Universidade Federal de Minas Gerais), disponível na base desde 2006.

O segundo, Ciência da Informação, uma das publicações com maior visibilidade no campo da Ciência da Informação no Brasil, trata de publicações quadrimestrais de trabalhos inéditos nessa área ou que apresentem resultados de estudos e pesquisas sobre as atividades do setor de informação em ciência e tecnologia. $O$ periódico Ciência da Informação tem tido atuação marcante no campo da Ciência da Informação e no setor de informação em C\&T, pela veiculação de contribuições significativas de especialistas nacionais e estrangeiros, pela abrangência de seu público que é constituído pela comunidade acadêmica de pesquisadores e profissionais, não só da Ciência da Informação como também de áreas correlatas.

A análise de citação, procedimento utilizado nas análises métricas, trata do número de citações recebidas por um documento, autor ou instituição. Apesar das controvérsias e debates, o uso deste procedimento possibilita a avaliação da visibilidade e do impacto de um documento, autor ou instituição, na produção do conhecimento científico da área ou tema sob análise, permitindo a visualização da sua frente de pesquisa.

Segundo Vanz e Caregnato (2003, p. 247), a "análise de citações identifica o perfil dos pesquisadores, mapeando suas áreas do conhecimento", além de, através do conjunto de referências de uma pesquisa, identificar "os pesquisadores cujos conceitos, métodos ou teorias serviram de inspiração, ou foram utilizados pelo autor no desenvolvimento de seu próprio trabaIho" (p. 248), evidenciando o comportamento dos pesquisadores.

Ainda a "análise de citações mapeia a comunicação científica" (Vanz; Caregnato, 200, p. 248), 
dá indicadores de como está ocorrendo a comunicação científica de uma área do conhecimento, contribuindo para a construção da rede de relações e da explicitação da comunicação e relacionamento entre seus pesquisadores.

Os autores destacam também que (Vanz; Caregnato, 2003, p. 251).

a análise de citações mede o impacto e a visibilidade de determinados autores dentro de uma comunidade científica, evidenciando quais 'escolas' do pensamento vigoram dentro das mesmas.

Em resumo, a análise de citação contribui para o entendimento de uma comunidade científica, identificando os pesquisadores com maior impacto na área, dando visibilidade às referências teóricas que sustentam a área, bem como seus conceitos, objetos e métodos.

Segundo Small (2004, p. 72),

quando os cientistas concordam quanto ao que constitui a literatura relevante a priori, incluindo o que é significante naquela literatura, eles estão de fato definindo as estruturas de suas comunidades.

Para um pesquisador, o pagamento (recompensa) pelo seu trabalho é o reconhecimento pelos seus pares e este vem (está associado) pela citação. Assim, a citação é a moeda de pagamento no mundo acadêmico, associada à idéia de unidade (átomo) de reconhecimento.

A "estrutura da ciência é gerada por padrões de co-reconhecimento" (Small, 2004, p. 71).

Desta forma (Small, 2004, p. 76),

se a norma de citação envolve um pagamento simbólico de débito intelectual, ela é, ao mesmo tempo, uma atribuição de significado para o texto citado e uma construção de seu significado. Documentos funcionam como símbolos conceituais para os autores citantes ao mesmo tempo que crédito ou descrédito simbólico está sendo conferido. Por extensão, quando documentos são cocitados, autores citantes estão atribuindo coreconhecimento bem como criando uma associação de significados

Small, um dos primeiros estudiosos no tema análise de cocitação, define a cocitação como a frequência com que duas referências da literatura são citadas juntas por uma literatura posterior. Ainda, segundo o autor, a cocitação pode ser usada para estabelecer o núcleo da literatura citada dentro de determinado tema ou área em particular. Segundo Callon, Courtial e Penan (1995), documentos citados conjuntamente são os fundamentos, enquanto que os artigos que operam com essas citações conjuntas indicam os pioneiros e contribuem para o desenvolvimento do paradigma que é explorado em todas as suas potencialidades.
Spinak considera que as cocitações indicam as relações e frequências dos pares de documentos que são citados conjuntamente por outros documentos, estendendo este conceito a revistas ou autores; observa que, em relação a documentos ou revistas, a unidade de análise são os documentos ou revistas, e no segundo caso são os autores individuais.

A premissa fundamental de análise de cocitação é que "quanto maior for a quantidade de vezes que dois documentos são co-citados conjuntamente, é maior a probabilidade de que sejam relacionados em conteúdo" (Spinak, 1996, p. 13).

Em síntese, dois documentos, revistas ou autores são cocitados quando ambos aparecem na lista de referências de um terceiro documento, revista ou autor.

\section{Ainda segundo Spinak (1996, p. 16),}

os grupos de co-citações representam a frente de investigação das diferentes áreas do conhecimento, tal como se mostram na literatura citada. Sem dúvida, esses agrupamentos podem representar tanto as redes cognitivas, como as redes sociais entre os investigadores. As redes de co-citações podem traduzir-se em mapas ou nós, onde os pontos denotam documentos e as linhas de união representam as relações de co-citações.

O autor em questão observa que a análise de cocitação é uma ferramenta limitada pela seleção inicial dos autores, que deverá ser adequada e representativa, observando que os autores mais citados, quer sejam os "clássicos", fazem parte, em geral, desta seleção.

O uso das cocitações como instrumento para a visibilidade de pesquisadores em determinado tema ou área torna possível a visualização da frente de pesquisa determinada, através da construção de redes sociais de comunicação científica.

Segundo Wasserman e Faust (1994, p. 9), "o termo 'rede social' se refere ao conjunto de atores e suas ligações entre eles". A análise de rede tem por objetivo modelar as relações entre os atores, a fim de retratar, descrever e representar a estrutura de um grupo.

O conceito de análise de rede social desenvolveu-se a partir de uma confluência da teoria social com a metodologia matemática, estatística e computacional. Ainda, segundo os autores em questão, entre os pilares da modelagem da análise de rede social encontra-se a exibição visual da estrutura do grupo por meio do sociograma, conceituado como (Wasserman; Faust, 1994, p. 10), 
figura na qual pessoas (ou mais geralmente, qualquer unidade social) são representadas como pontos em espaços bidimensionais, e relacionamentos entre pares de pessoas são representados por linhas ligando os pontos correspondentes.

Destaque-se que a análise de rede social está preocupada em entender as ligações entre entidades sociais e as implicações destas ligações, consistindo em um conjunto finito (ou conjuntos de atores) e a relação (ou relações) definida entre eles.

\section{Metodologia}

O procedimento de pesquisa adotado para identificação, análise e avaliação dos dados foi 0 levantamento dos números publicados on-line nos periódicos Perspectivas em Ciência da Informação e Ciência da Informação. O primeiro, com apenas três anos de publicação on-line, envolveu um total de 68 artigos, sendo que somente 11 tratam de Estudos Métricos, ou seja, $16,2 \%$. No segundo, com 11 anos de publicações on-line, foram analisados 320 artigos, sendo que somente 42 tratam do tema em questão, ou seja, $13,1 \%$. Trabalhou-se, inicialmente, com o universo de 388 artigos, sendo 53 (13,7\%, entre os dois periódicos) os artigos que tratam do tema Estudos Métricos.

Levantaram-se os autores mais citados, através do software Excel. Em um total de 1514 citações, foram citados 888 autores, obtendo-se uma média de 1,7 citações por autor. Deste grupo, 35 autores foram citados pelo menos seis vezes em um total de 318 citações, dados estes apresentados em tabela no corpo do trabalho.

Considerando que montada a matriz de cocitação para estes 35 autores mais citados, a rede de cocitação ficou com pouca visibilidade, devido a muitos elos com baixa frequência, o que dificultou a visualização da real frente de pesquisa. Optou-se por recortar ainda mais o ranking obtido, a fim de se obter uma visualização mais clara, estabelecendo-se, para representação, uma matriz de $18 \times 18$ autores, quadrada e simétrica, com autores que receberam pelo menos oito citações. Este procedimento foi baseado na assertiva de Spinak, pesquisador citado, que afirma que a análise de cocitação é limitada pela seleção inicial dos autores, que deverá ser adequada e representativa.

Foi utilizado o software Pajek, a fim de mapear e visualizar a frente de pesquisa, a partir dos autores mais citados (pelo menos oito citações) e que foram cocitados de forma pareada.

\section{Apresentação e análise dos dados}

Em um total de 1514 citações, 35 autores foram citados pelo menos seis vezes em um total de 318 citações (Tabela I), que correspondem a $21 \%$ das citações. O restante aparece com 5 ou menos de 5 citações e a grande maioria (658) dos autores é citada apenas uma vez, o que sugere muitos pesquisadores produzindo na área, porém com baixa frequência de citação e ainda pouco reconhecidos.

Por outro lado, entre os pesquisadores com alta frequência de citação, aparecem os "clássicos" mais citados, tais como: Ronald Rousseau, (Bélgica), com 22 citações; Eugene Garfield (EUA), com 19 citações; Peter Ingwersen (Dinamarca), Wolfgang Glänzel (Bélgica) e MaciasChapula (México), com 15 citações; Ernesto Spinak (Uruguai), Moya-Anegon (Espanha) e Derek Solla Price (EUA), com 12 citações. Destaque seja feito para a brasileira Suzana Pinheiro Machado Muller, com 11 citações, colocandose em $9^{a}$ posição na lista dos mais citados.

Em relação à origem dos pesquisadores mais citados, aparecem, em âmbito internacional, os E.U.A, Espanha, França e Reino Unido de forma mais frequente. Destaquem-se os E.U.A. e a Espanha, países pioneiros na área de Bibliometria e Cienciometria e com densa produtividade atual no tema Estudos Métricos. O Brasil aparece com o maior número de pesquisadores mais citados, mostrando assim a visibilidade dos pesquisadores brasileiros, mesmo em se tratando de uma pesquisa feita em periódicos do próprio país.

Observa-se ainda que os pesquisadores Rousseau e Macias-Chapula, além de alta citação, apresentam-se também como os autores com maior inserção e penetração no tema, considerando que os mesmos são os autores citados em maior número de artigos (23\%). Também Price, Glänzel, Spinak, Garfield, Mueller, Quoniam e Meadows aparecem com significativa penetração no tema, uma vez que foram citados entre $15 \%$ e $21 \%$ dos artigos analisados. Por outro lado, Thelwall aparece citado somente em um artigo entre os analisados, porém com alta citação (nove trabalhos distintos do autor).

Apresenta-se, a seguir, a rede de cocitação construída com os primeiros 18 autores mais citados. Esclarece-se que as áreas dos círculos se referem à frequência com que os autores foram citados e a espessura dos segmentos de reta, à intensidade de cocitação entre os pares. 


\begin{tabular}{|c|c|c|}
\hline Autores (país) & $\begin{array}{l}N^{0} \text { de cita- } \\
\text { ções rece- } \\
\text { bidas }\end{array}$ & $\begin{array}{c}\mathrm{N}^{\circ} \text { de } \\
\text { artigos } \\
\text { em que } \\
\text { foi citado }\end{array}$ \\
\hline Rousseau, R. (Bélgica) & 22 & 12 \\
\hline Garfield, E. (EUA) & 19 & 8 \\
\hline Ingwersen, P. (Dinamarca) & 15 & 4 \\
\hline Glänzel, W. (Bélgica) & 15 & 9 \\
\hline Macias-Chapula, C. A. (México) & 15 & 12 \\
\hline Spinak, E. (Uruguai) & 12 & 9 \\
\hline Moya-Anegón, F. (Espanha) & 12 & 3 \\
\hline Price, D. J. de S. (EUA) & 12 & 11 \\
\hline Mueller, S. P.M. (Brasil) & 11 & 9 \\
\hline Quoniam, L. (França) & 9 & 8 \\
\hline Thelwall, M. (Reino Unido) & 9 & 1 \\
\hline Wormell, I. (Suécia) & 9 & 5 \\
\hline Courtial, J.P. (França) & 8 & 3 \\
\hline Velho, L. (Brasil) & 8 & 6 \\
\hline Braga, G. M. (Brasil) & 8 & 7 \\
\hline Meadows, A. J. (Reino Unido) & 8 & 8 \\
\hline Moed, H. F. (Holanda) & 8 & 6 \\
\hline Vinkler, P. (Hungria) & 8 & 4 \\
\hline Frereira, M. C. G. (Brasil) & 7 & 4 \\
\hline Jiménez-Contreras, E. (Espanha) & 7 & 2 \\
\hline Katz, J. S. (EUA) & 7 & 5 \\
\hline Newman, M. E. J. (EUA) & 7 & 4 \\
\hline Noronha, D. P. (Brasil) & 7 & 6 \\
\hline Pecegueiro, C. M. P. A. (Brasil) & 7 & 7 \\
\hline Pinheiro, L. V. R. (Brasil) & 7 & 5 \\
\hline Tague-Sutcliffe, J. (Canadá) & 7 & 7 \\
\hline Vanti, N. A .P. (Brasil) & 6 & 6 \\
\hline Cronin, B. (EUA) & 6 & 4 \\
\hline Cunha, M. B. da. (Brasil) & 6 & 3 \\
\hline Herrero Solana, V. (Espanha) & 6 & 2 \\
\hline Martin, B.R. (Reino Unido) & 6 & 5 \\
\hline Meneghini, R. (Brasil) & 6 & 4 \\
\hline Persson, O. (Suécia) & 6 & 5 \\
\hline Robredo, J. (Brasil) & 6 & 2 \\
\hline Rostaing, H. (França) & 6 & 4 \\
\hline
\end{tabular}

TABELA I. Autores mais citados (até 6 citações).

Destacam-se, em um primeiro momento, na região mais densa da rede, os seguintes pesquisadores: Rousseau, Garfield e Glänzel. Nesta frente é contemplado também MaciasChapula, com alta frequência de cocitações, associado a Rousseau, além de Spinak, Moed e Wormell, associados a este núcleo com significativa frequência de cocitação, apesar de os dois últimos, no ranking de citações, não terem sido contemplados nos primeiros lugares. Destaque-se, ainda, que os autores Rousseau, Garfield e Glänzel possuem os maiores números de conexões com os outros autores da rede, sendo que o primeiro é cocitado com $94 \%$ dos autores e os outros dois, com $88 \%$ deles. Vale lembrar que as posições centralizadas não são características dos autores, mas refletem aspectos relacionais desta rede em particular.

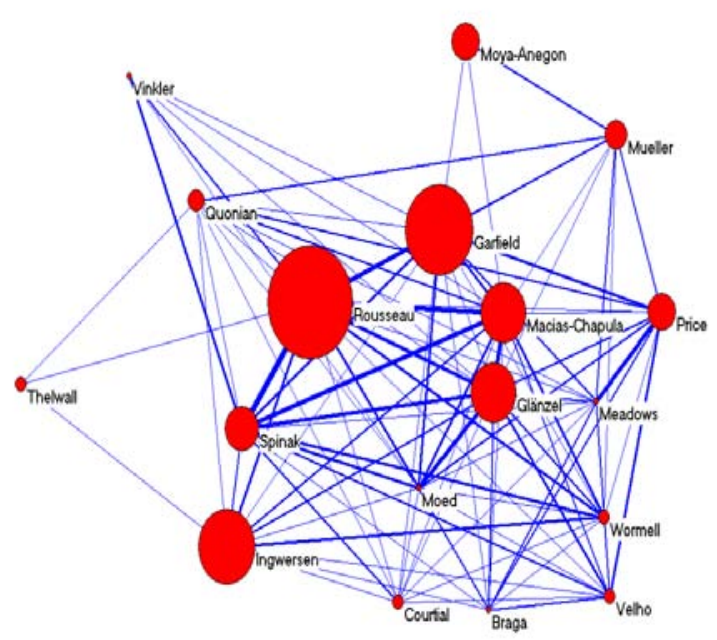

Figura I. Rede de co-citações dos autores com pelo menos 8 citações.

Outros autores com alta cocitação são Price e Meadows. Destaque-se ainda as brasileiras Velho, Braga e Mueller, com número alto de cocitações, inclusive com o núcleo principal.

É importante ainda enfatizar outros autores, com participação mais modesta na rede, como Moya-Anegon e Thelwall, porém de visibilidade internacional, especialmente o primeiro que compõe o grupo espanhol que pesquisa as questões métricas.

Foi calculada a densidade da rede, medida de coesão, através da razão entre as conexões presentes na rede (100) e o número total de conexões possíveis (153), resultando em 0,65, significando que $65 \%$ das conexões possíveis estão presentes na rede, o que aponta uma rede relativamente densa.

Assim, a rede apresenta-se de forma não fragmentada, porém com alguns autores de presença mais consistente e que determinam a maior visibilidade da frente de pesquisa.

\section{Considerações finais e recomendações}

Como considerações finais, observa-se que o tema estudos métricos é pouco consolidado no Brasil, com modesta produção científica, apesar do comparecimento de pesquisadores brasileiros na frente de pesquisa. 
Destaque-se também o caráter multidisciplinar do tema, fato que acarreta uma pulverização grande de autores advindos das mais diferentes áreas de conhecimento, que passaram a se preocupar nos últimos anos com questões cienciométricas. Os países pioneiros em estudos métricos compõem o "core" desta frente.

Recomendam-se estudos similares em diferentes temáticas na área, em outras áreas e em diferentes bases, para que se possa, de forma abrangente e plena, ter a visibilidade dos pesquisadores que atuam nas diferentes áreas do conhecimento, tanto em âmbito nacional, como internacional. Além disso, sugere-se que pesquisas que mapeiam redes de produção em determinados temas sejam abordadas também de forma contextual e qualitativa, a fim de se esclarecer as associações entre os autores cocitados, inclusive aquelas relativas à similaridade e complementaridade em seus referenciais teóricos ou metodológicos.

\section{Referências}

Callon, Michel; Courtial, Jean-Pierre; Penan, Hervé (1995). Cienciometría. La medición de la actividad científica: de la bibliometría a la vigilancia tecnológica. Astúrias: Ediciones Trea, S. L., 1995.

Graeml, Alexandre Reis; Macadar, Marie Anne; Guarido Filho, Edson R.; Rossoni, Luciano (2008). Redes sociais e intelectuais em ADI: uma análise cientométrica do período 1997-2006. // Anais do 32th Encontro Nacional da Associação Nacional de Pós-Graduação e Pesquisa em Administração (EnANPAD), 32, Rio de Janeiro, 2008. Anais.... Rio de Janeiro: ANPAD, 2008.

Liberatore, Gustavo; Herrero-Solana, Victor; Guimarães, José Augusto Chaves (2007). Análise bibliométrica do periódico brasileiro Ciência da Informação durante o período 2000-2004. // Brazilian Journal of Information Science, 1:2 (jul./dez. 2007) 3-21. http://www.bjis. unesp.br/pt/.

Olmeda Gómez, Carlos; Perianez- Rodriguez, Antonio; Ovalle-Perandones, Maria Antonia (2008). Estructura de las redes de colaboración científica entre las universidades españolas. // Ibersid. (2008) 129-140.

Pinheiro, Liliane Vieira; SILVA, Edna Lúcia da (2008). As redes cognitivas na Ciência da Informação brasileira: um estudo nos artigos científicos publicados nos periódicos da área. // Ciência da Informação. 37: (2008) 75-87.

Pinto, Adilson Luis; Barquín, Beatriz-Ainhize Rodríguez.; González, José Antonio Moreiro (2006). Análisis de citación de la revista Ciência da Informação del lbict. // Ciência da Informação. 35:3 (set./dez.2006) 153-165.

Small, Henry (1973). Co-citacion in the Scientific Literature: A New Measure of the Relationship Between Two Documents. // Journal of de American Society for Information Science. 24:4 (Jul/Aug 1973) 265-269.

Small, Henry (2004). On the shoulders of Robert Merton: towards a normative theory of citation. // Scientometrics. 60:1 (2004) 71-79.

Spinak, Ernesto. (1996). Dicionario enciclopédico de Bibliometria, Cienciometria e Informetria. Venezuela: UNESCO, 1996.

Vanz, Sandra Andréa de Souza; Caregnato, Sônia, Elisa (2003). Estudos de citação: uma ferramenta para enten- der a comunicação científica. // Em Questão. 9:2 (jul./dez. 2003) 295-307.

Wasserman, Stanley; Faust, Katherine (1994). Social network analysis: methods and applications. Cambridge: Cambridge University Press, 1994. 